www.jmscr.igmpublication.org

Index Copernicus Value: 79.54

ISSN (e)-2347-176x ISSN (p) 2455-0450

crossref DOI: https://dx.doi.org/10.18535/jmscr/v7i6.187

\title{
Do Entamoeba histolytica commonly infect children?
}

\author{
Authors \\ Mona Mohamed Tolba ${ }^{*}$, Omneya Magdy Omar², \\ Ahmed Abdelfattah Abdelaziz Aboelhassan ${ }^{2}$, Magdy Abd El fattah Ramadan ${ }^{2}$ \\ ${ }^{1}$ Department of Parasitology, Medical Research Institute, University of Alexandria, Egypt \\ ${ }^{2}$ Department of Pediatrics, Faculty of Medicine, University of Alexandria, Egypt \\ *Corresponding Author \\ Mona Mohamed Tolba
}

\begin{abstract}
Introduction: Gastrointestinal infections are commonly present in developing countries specially tropical regions. Infections of the gastrointestinal tract can be caused by viruses, bacteria, protozoa, helminths or fungi. Among pathogenic intestinal protozoa causing diarrhea, Entamoeba histolytica and Giardia lamblia are the most frequently encountered in both industrialized and developing countries. Cryptosporidium parvum is now recognized as an important cause of diarrhea in young children and immunocompromized adults.

Methods: Hundred and six children with diarrhea were enrolled in the study and stool samples were collected and examined for parasitic infections. The aim of this study is to assess the true infection by pathogenic E. histolytica in children attending emergency department of Alexandria university children's hospital with diarrhea. Comparison between microscopic examination and antigen detection using ELISA in diagnosing and confirming of E. histolytica infection was done.

Results: E. histolytica/dispar was present in 7 out of 106 cases (6.6\%) by microscopic examination and 3 out of 106 cases (2.8\%) by ELISA technique for E.histolytica/dispar stool antigen and only lout of 106 cases (0.94\%) by ELISA technique specific for E.histolytica stool antigen.

Conclusions: E. histolytica is one of the uncommon causes of diarrhea in children and should be diagnosed using ELISA technique specific for E. histolytica stool antigen which is more specific and sensitive than stool microscopic examination.
\end{abstract}

Keywords: diarrhea; Entamoeba histolytica; diagnosis; microscopy; ELISA.

\section{Introduction}

Diarrheal diseases are the second leading cause of death in children under five years old. In developing countries, children under three years old experience an average three episodes of diarrhea every year. As a result, diarrhea is a major cause of malnutrition, and malnourished children are more likely to fall ill from diarrhea. ${ }^{(1)}$
Diarrhea is due to the wide variability of pathogens that can be bacterial, viral and parasitic. In developed countries, viral pathogens are the major cause. ${ }^{(2,3)}$ In developing countries, viral pathogens, enteric bacteria, and parasites are more predominant due to poor personal hygiene and sanitation $^{(2,4)}$

Parasitic diseases are common generally worldwide. A wide scope of protozoa 
contaminates children intestinal tract. Their prevalence is higher in areas with low standards of sanitation and cleanliness. E. histolytica, $G$. lamblia, and $C$. parvum are determined the main important diarrhea-causing protozoa around the world. ${ }^{(5)}$

E. histolytica infections are prevalent in the developing world with tropical climates. In some tropical nations, prevalence rates exceed half.In developed countries, the general prevalence of $E$. histolytica has been evaluated to be around 4\%. It happens in foreigners, travellers who travel to endemic regions. ${ }^{(6)}$

About $10 \%$ of yearly infected patients are symptomatic. Entamoeba dispar (E. dispar), a different non-pathogenic species which is identical in morphology to E. histolytica, produce $90 \%$ of the 500 million new amoebic infections every year. ${ }^{(7)}$

The life cycle of E. histolyticais simple that consists of an infective cyst form and an amoeboid trophozoite stage. The cyst measures $10-15 \mu \mathrm{m}$ in diameter and contain 1 to 4 nuclei, depending on its level of maturation. The trophozoite is $10-50 \mu \mathrm{m}$ in diameter and contains a single nucleus. ${ }^{(8)}$

The clinical picture of amoebiasis ranged from asymptomatic to fulminant colitis and peritonitis to extraintestinal amebiasis, for example, an amebic liver abscess. Amebiasis is more severe in younger children. $90 \%$ of E histolytica infections are asymptomatic, the infection is self-limited but may be repeated. It is not likely to distinguish between $E$ histolytica and $E$ dispar on clinical basis; only antigen detection tests can reveal this difference. $^{(9)}$

The diagnosis of intestinal protozoa is determined mainly by microscopic recognition of the various parasite stages in stool, duodenal fluid, or small intestine biopsy specimens. Other detection approaches such as serology, immune-diagnosis or molecular diagnosis could be beneficial. ${ }^{(10)}$

Microscopic examination: Non- permanent staining; stool specimens should be identified either without stain or with a stain by methylene blue or Lugol's iodine which makes the nucleus successfully detectable. The presence of chromatid bodies in the cyst is similar to in the wet mount preparations. ${ }^{(11)}$ Permanent staining; several stains, including Giemsa, Wright's and trichrome can be used perfectly. Trichrome staining of permanent smears has been recommended for detection of $E$. histolytical $E$. dispar. $^{(12)}$

Culturing E. histolytica from stool or liver abscess samples is mostly inadequate and not beneficial in laboratory practice. ${ }^{(13)}$ It is mainly a research tool rather than a diagnostic one. ${ }^{(14)}$

Antibody detection; widespread different antibody analyses for recognition of $E$. histolytica antibodies in serum are commercially available. In regions where the infection is endemic the incompetence of serological tests to differentiate past from recent infection creates a diagnostic difficulty. ${ }^{(15)}$

Antigen Detection is a quick technique for the direct detection of antigenic components of parasites in different body fluids or tissues for rapid and definite diagnosis of acute infection. Antigen recognition tests examples are: Enzymelinked immunosorbent assay (ELISA), direct fluorescent antibody (DFA) and immunochromatography (IC). ${ }^{(16)}$

Molecular-based technology offers sensitivity and specificity for amoebiasis diagnosis that challenges that of antigen detection. Detection of E. histolytica can be done from different clinical samplings, such as stool, liver abscess aspirate, and tissues. ${ }^{(17)}$

The aim of this study is to assess the true infection by pathogenic $E$. histolytica in children attending emergency department of Alexandria university children's hospital with diarrhea. Comparison between microscopic examination and antigen detection using ELISA in diagnosis and confirmation of E. histolytica infection was done.

\section{Methods}

The study was conducted on 106 children aged from 1 to 5 years attending the emergency 
department of Alexandria University Children's Hospital with acute diarrhea for less than 14 days with or without blood in stool.

Stool samples were collected from patients and homogenized by thorough mixing immediately after delivery to the laboratory. One part of the sample was kept in a labelled clean tube without preservation and stored at $-20^{\circ} \mathrm{C}$ for ELISA. Another part was subjected to Formol-ethyl acetate concentration technique and modified acid-fast stain. $^{(18,19)}$

\section{Results}

1- Microscopic examination of the studied group

\section{- Wet mount examination}

After concentration and wet mount examination; 66 children $(62.00 \%)$ were negative for parasitic infections, 27 children (25\%) had Blastocystis hominis, 7 children $(6.6 \%)$ had E. histolytical dispar, 6 children $(5.6 \%)$ had E. coli, 4 children (3.7\%) had Dientamoeba fragilis (D. fragilis) and only one child $(0.94 \%)$ showed Giardia lambilia. Five children $(4.71 \%)$ had combined infection with Blastocystis hominis with E. coli, 3 children (2.83\%) had Blastocystishominis with D. fragilis and only one child (0.9\%) with Blastocystis hominis with oxyuris. (Figure 1)

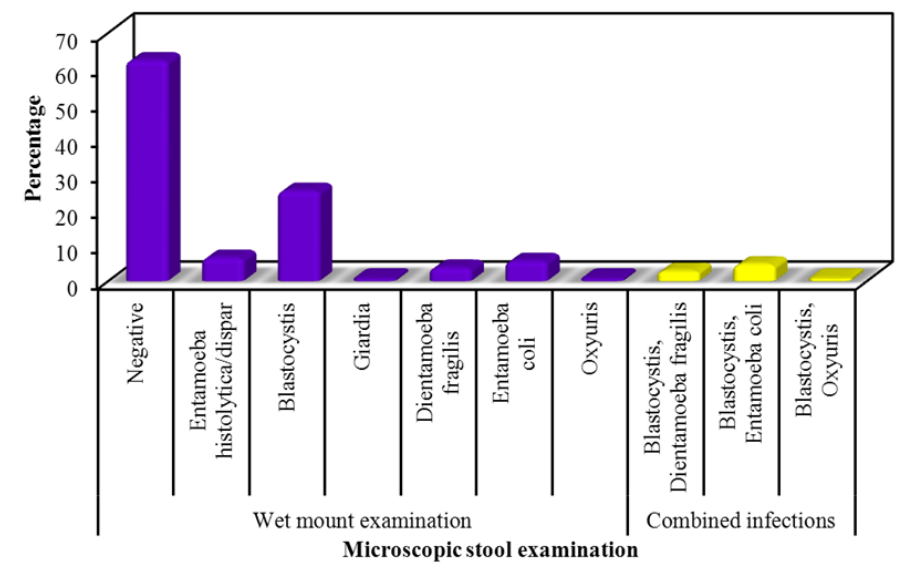

Figure (1): Microscopic wet mount stool examination of the examined 106 children.

- Microscopic examination by Modified Zihl-Nelsen stain (MZN stain)

Using MZN stain, 2 children (1.88\%) had $C$. parvum, one child had Cyclospora cayatenensis
$(0.94 \%)$ and 103 children $(97.16 \%)$ were negative. (Figure 2)

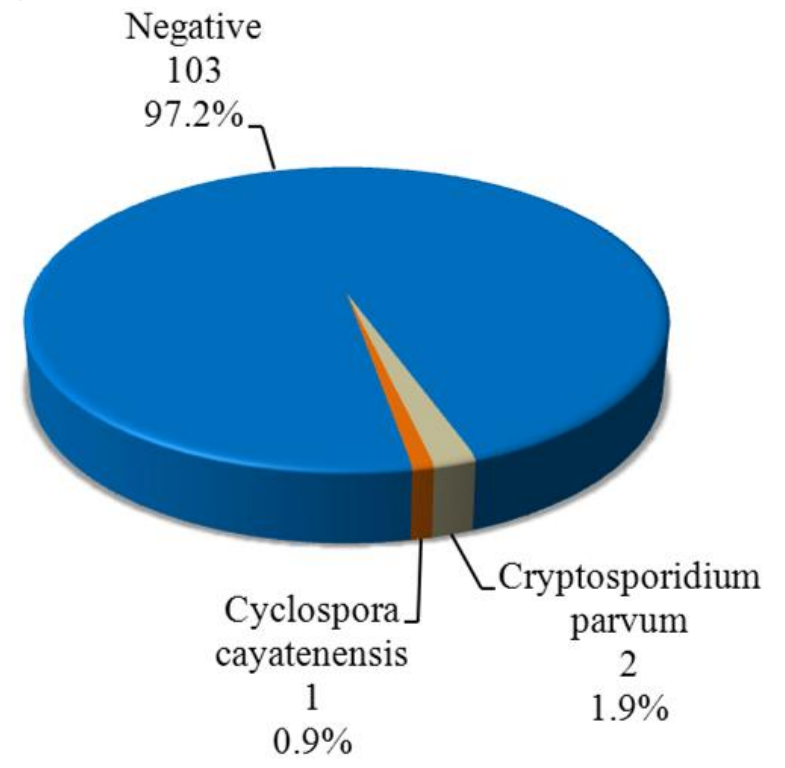

Figure (2): Microscopic examination by MZN stain of the studied children.

\section{2- ELISA techniques}

ELISA for E.histolytica/dispar was done for all stool samples, 3 children $(2.83 \%)$ had positive results and 103 children $(97.17 \%)$ were negative. Regarding ELISA specific for E.histolytica only, one child $(0.94 \%)$ had positive result and 105 children $(99.06 \%)$ were negative.

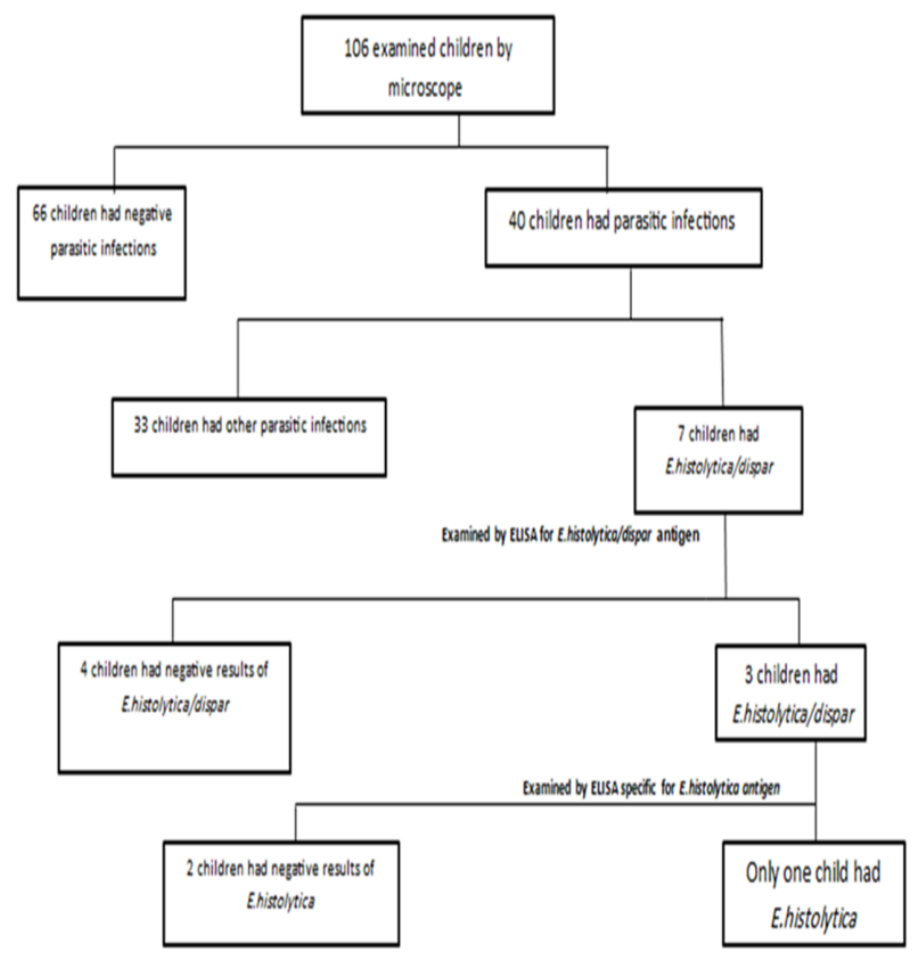

Figure 3: Flow chart of the study results. 
Agreement analysis between microscopic stool examination for E. histolytica and ELISA technique specific for E.histolytica: ${ }^{(20)}$

Comparing the results of the 106 children examined by ELISA specific for E. histolytica with their results by microscopic examination, it revealed that only one child gave positive concordant results. By analysis of the discordant results, 6 children were positive by microscopic examination and negative by ELISA specific for E. histolytica. Statistical analysis revealed a Kappa index of 0.237 showing fair agreement between both techniques in diagnosing $E$. histolytica infection. (Table 1)

\begin{tabular}{|c|c|c|c|c|}
\hline \multirow{2}{*}{\multicolumn{2}{|c|}{ Agreement }} & \multicolumn{2}{|c|}{$\begin{array}{l}\text { ELISA specific for } \\
\text { E. histolytica }\end{array}$} & \multirow{3}{*}{$\begin{array}{c}\text { Total } \\
99\end{array}$} \\
\hline & & Negative & Positive & \\
\hline \multirow{2}{*}{$\begin{array}{l}\text { Microscopic } \\
\text { examination }\end{array}$} & Negative & 99 & 0 & \\
\hline & Positive & 6 & 1 & 7 \\
\hline \multicolumn{2}{|l|}{ Total } & 105 & 1 & 106 \\
\hline
\end{tabular}

Kappa index $=0.237, \mathrm{p}<0.001$ fair agreement

\section{Discussion}

Parasitic infections, and in particular of protozoan causes, represent a major, but often ignored threat to the public health worldwide. They are the most widespread infections in developing countries with children being the main liable population. ${ }^{(21)}$ In those countries, poor sanitary conditions and inaccessibility of effective water treatment have sustained conditions for their transmission. Pathogenic intestinal protozoa represent the main reason for gut illness with great impact. ${ }^{(22)}$ Assessment of the burden of illness is often problematic by the shortage of reliable data due to under-diagnosis and absence of monitoring programs ${ }^{(23)}$

Although great advances has been made in laboratory diagnosis, laboratories in developing countries continue to depend on ova and parasite microscopic examination as the main approach for recognition of parasites being comparatively cheap and suitable for resource-limited countries. However, accurate identification of parasites is mostly dependent on the level of skills and expertise of the laboratory technicians, and therefore its sensitivity and specificity vary from one laboratory to another. For this reason, alternative acceptable approaches for accurate identification of different protozoa have been suggested including immunoassays and PCR. ${ }^{(24)}$

The purpose of the present study was to evaluate the frequency of parasitic infections as one of the significant reasons of diarrhea in children and recognition of pathogenic E. histolytica to reveal its true burden as a cause of diarrhea in children. Comparison between microscopic examination and antigen detection using ELISA in determining E. histolytica infection was done.

This study was done on 106 children attending the emergency department of Alexandria University Children's Hospital with acute diarrhea with or without blood in stool aged from 1 year to 5 years old.

Regardingthe prevalence of different parasitic infections in children detected by wet mount microscopic examination. The current study showed that the most common parasite was $B$. hominis $27(25 \%)$ children, followed byE. histolytica/dispar 7 children (6.6\%) and the least was D.fragilis 4 children (3.7\%).MZN stainrevealed $C$. parvum in 2 children (1.9\%) and C.cayatenensisin only one child $(0.9 \%)$.

These results are in agreement with Nimri et al. ${ }^{(25)}$, showing that $B$. hominis was detected in 63 (25\%) out of 250 stool specimens from preschool children in northern Jordan diagnosed by wet mount microscopic examination. 38 samples (15\%) contained B. hominis in the absence of other pathogens. The other $25(10 \%)$ had other pathogenic parasites, bacteria, or rotavirus in the same specimen. Monib et $\mathrm{al},{ }^{(26)}$ reported that the most prevalent parasite wasG. lamblia (10.4\%) followed by $E$. coli $(2.7 \%)$ using wet mount microscopic examination and Cryptosporidium $(2.3 \%)$ by MZN stain.

On the other hand,according to El-Sehry et al. $2017^{(27)}$, the incidence of parasitic infections and its effect on the health status of 300 children in El Mahalla El Kobra was assessed. It was observed that about 225 studied children $(73.2 \%)$ were infected by E. histolytica compared to 60 children 
(20\%) infected by oxyuris, only 5 children (1.6\%) were infected by $G$. lamblia using wet mount microscopic examination.

Regarding agreement between microscopic examination for E. histolytica/dispar and ELISA specific for E. histolytica; by comparing the results of the 106 children examined by ELISA specific for E. histolytica with their results after microscopic examination, it was revealed that only one sample gave positive concordant result. Statistical analysis revealed a Kappa index of 0.237 showing fair agreement between both techniques. This indicates that ELISA specific for E. histolyticais more sensitive and specific than microscopic examination in the diagnosis of $E$. histolytica.

Hegazi et al. 2013 ${ }^{(28)}$, who studied the prevalence and characters of E.histolytica infection in 738 Saudi children attending 2 main hospitals at south Jeddah with diarrhea. 120cases (20\%) were diagnosed E. histolytica of all cases. Confirmation for E. histolytica infection using E. histolytica antigen detection test that demonstrated enhanced sensitivity and specificity for recognition of $E$. histolytica infection than microscopic examination of stool samples.

According to Delialioglu et al. 2008 ${ }^{(29)}$, stool samples from 272 children with diarrhea in the area of Mersin, Turkey, were studied for the occurrence of E.histolytical E. dispar microscopically and forE. histolytica antigen using the ELISA method. Microscopic examination reported 70 children $(25.7 \%)$ had E.histolotica/dispar and ELISA test showed 29 children (10\%). E. histolytica-specific ELISA was positive in $21(7.72 \%)$ and $E$. dispar positive in 8 (2.94\%) samples. Detection of true E. histolytica infection allows actual cases of amoebiasis to be identified and cured, and over-treatment of children with E. dispar, which is the nonpathogenic species, to be stopped.

In conclusion, E.histolytica is an uncommon cause of diarrhea in children. Microscopic analysis cannot differentiate between the pathogenic E.histolotica and non-pathgenic E.dispar and must be confirmed by ELISA technique for accurate diagnosis to avoid unnecessary drug intake. Increase competency of technicians and chemists by regular training for identification of different parasites in every lab is mandatory.

\section{References}

1. Fontaine $\mathrm{O}$, Kosek $\mathrm{M}$, Bhatnagar $\mathrm{S}$, Boschi-Pinto C, Chan KY, Duggan C, et al. Setting research priorities to reduce global mortality from childhood diarrhea by 2015. PLoS Med 2009; 6(3):e1000041.

2. Massoud NM, Said DE, El-Salamouny AR. Prevalence of Cyclospora cayetanensis among symptomatic and asymptomatic immune-competent children less than five years of age in Alexandria, Egypt. Alex J Med 2012; 48(3):251-9.

3. Mustafa H, Palombo EA, Bishop RF. Epidemiology of astrovirus infection in young children hospitalized with acute gastroenteritis in Melbourne, Australia, over a period of four consecutive years, 1995 to 1998. J Clin Microbiol 2000; 38(3):1058-62.

4. Ono K, Rai SK, Chikahira M, Fujimoto T, Shibata H, Wada Y, et al. Seasonal distribution of entero-pathogens detected from diarrheal stool and water samples collected in Kathmandu, Nepal. Southeast Asian J Trop Med Public Health 2001; 32(3):520-6.

5. Stark D, Van Hal S, Fotedar R, Butcher A, Marriott D, Ellis J, et al. Comparison of stool antigen detection kits to PCR for diagnosis of amebiasis. J Clin Microbiol 2008; 46(5):1678-81.

6. El-Dib NA. Entamoeba histolytica: an Overview. Curr Trop Med Rep 2017; 4(1):11-20.

7. Field M, Rao MC, Chang EB. Intestinal electrolyte transport and diarrheal disease. N Engl J Med 1989; 321(12):800-6.

8. Davis $\mathrm{PH}$, Zhang Z, Chen M, Zhang X, Chakraborty S, Stanley Jr SL. 
Identification of a family of Bsp-A like surface proteins of Entamoeba histolytica with novel leucine rich repeats. Mol Biochem Parasitol 2006; 145(1):111.

9. Stanley Jr SL. Amoebiasis. Lancet 2003; 361(9362):1025-34.

10. Macpherson C, Gottstein B, Geerts S. Parasitic food-borne and water-borne zoonoses. Rev Sci Tech 2000; 19(1):24058.

11. Choudhuri G, Rangan M. Amebic infection in humans. Indian $J$ Gastroenterol 2012; 31(4):153-62.

12. Ravdin J, Petri W. Entamoeba histolytica (amebiasis). In: Mandell R, Douglas G, Bennett J (eds). Principles and practice of infectious diseases. New York, NY: Churchill Livingstone; 1990. 2036-49.

13. Evangelopoulos A, Spanakos G, Patsoula E, Vakalis N, Legakis N. A nested, multiplex, PCR assay for the simultaneous detection and differentiation of Entamoeba histolytica and Entamoeba dispar in faeces. Ann Trop Med Parasitol 2000; 94(3):233-40.

14. Khairnar K, Parija SC. Detection of Entamoeba histolytica DNA in the saliva of amoebic liver abscess patients who received prior treatment with metronidazole. J Health Popul Nutr 2008; 26(4):418.

15. Sargeaunt P. The reliability of Entamoeba histolytica zymodemes in clinical diagnosis. Parasitol Today 1987; 3(2):403.

16. Schwartz B, Mawhorter S, Practice AIDCo. Parasitic infections in solid organ transplantation. Am J Transplant 2013; 13(s4):280-303.

17. Singh A, Houpt E, Petri WA. Rapid diagnosis of intestinal parasitic protozoa, with a focus on Entamoeba histolytica. Interdiscip Perspect Infect Dis 2009; 2009:547090.
18. Garcia LS. Diagnostic Medical Parasitology. 6th ed. Washington, DC.: ASM Press; 2016. pp.26-76.

19. Haque R, Neville LM, Hahn P, Petri W. Rapid diagnosis of Entamoeba infection by using Entamoeba and Entamoeba histolytica stool antigen detection kits. J Clin Microbiol 1995; 33(10):2558-61.

20. Field A. Discovering Statistics Using IBM SPSS Statistics. 4th ed. London, California, New Delhi: SAGE Publications Ltd; 2013.

21. Osman M, El Safadi D, Cian A, Benamrouz S, Nourrisson C, Poirier P, et al. Correction: Prevalence and Risk Factors for Intestinal Protozoan Infections with Cryptosporidium, Giardia, Blastocystis and Dientamoeba among Schoolchildren in Tripoli, Lebanon. PLoS Negl Trop Dis 2016; 10(4):e0004643.

22. Ajjampur SS, Gladstone BP, Selvapandian D, Muliyil JP, Ward H, Kang G. Molecular and spatial epidemiology of cryptosporidiosis in children in a semiurban community in South India. J Clin Microbiol 2007; 45(3):915-20.

23. Fletcher SM, Stark D, Harkness J, Ellis J. Enteric Protozoa in the Developed World: a Public Health Perspective. Clin Microbiol Rev 2012; 25(3):420-49.

24. Kurt O, Demirel M, Ostan I, Sevil NR, Mandiracioglu A, Tanyuksel $\mathrm{M}$, et al. Investigation of the prevalence of amoebiasis in Izmir province and determination of Entamoeba spp. using PCR and enzyme immunoassay. New Microbiol 2008; 31(3):393.

25. Nimri LF. Evidence of an epidemic of Blastocystis hominis infections in preschool children in northern Jordan. J Clin Microbiol 1993; 31(10):2706-8.

26. Monib M, Hassan A, Attia R, Khalifa M. Prevalence of intestinal parasites among children attending Assiut University 
Children's Hospital, Assiut, Egypt. J Adv Parasitol 2016; 3(4):125-31.

27. El-Sehry NM, Fouda LM, Hassan L. Prevalence of parasitic Infections and its Effect on the Health Status of Primary School Children. IOSR J Nurs Health Sci 2017; 6(4):41-52.

28. Hegazi MA, Patel TA, El-Deek BS. Prevalence and characters of Entamoeba histolytica infection in Saudi infants and children admitted with diarrhea at 2 main hospitals at south Jeddah: a re-emerging serious infection with unusual presentation. Braz J Infect Dis 2013; 17(1):32-40.

29. Delialioglu N, Aslan G, Ozturk C, Ozturhan H, Şen S, Emekdas G. Detection of Entamoeba histolytica antigen in stool samples in Mersin, Turkey. J Parasitol 2008; 94(2):530-2. 